\title{
POSITIVE CURVATURE, PARTIAL VANISHING THEOREMS AND COARSE INDICES
}

\author{
JOHN ROE
}

\section{INTRODUCTION}

Let $M$ be a complete Riemannian manifold and let $D$ be a generalized Dirac operator acting on sections of a Clifford bundle $S$ over $M$. It is well-known (see for example [1]) that there is a Weitzenbock formula

$$
D^{2}=\nabla^{*} \nabla+R,
$$

where $R$ is a certain self-adjoint endomorphism of $S$ constructed out of the curvature. (For example, in the classical case of the Dirac operator associated to a spin-structure, $R$ is pointwise multiplication by $\frac{1}{4}$ times the scalar curvature [4]).

The author's coarse index theory associates to $D$ an index that lies in the $K$-theory of the "translation $C^{*}$-algebra" $C^{*}(M)$. As in the classical case, the index vanishes if the curvature operator is uniformly bounded below by a positive constant. In [7, Proposition 3.11] this statement is generalized as follows. Suppose that there is a subset $Z \subseteq M$, such that for some constant $a>0$ one has $R_{x} \geqslant a^{2} I$ (as self-adjoint endomorphisms of $S_{x}$ ) for all $x \notin Z$ - we will then say that the operator $R$ is uniformly positive outside $Z$. Then the index of $D$ lies in the image of the map

$$
K_{*}\left(C^{*}(Z)\right) \rightarrow K_{*}\left(C^{*}(X)\right),
$$

where $Z$ is considered as a metric subspace of $X$. In particular, if the curvature is uniformly positive outside a compact set $Z$ (so that $C^{*}(Z)$ is the compact operators), one recovers the result of Gromov and Lawson [1, Chapter 3] that $D$ has an index in the ordinary Fredholm sense.

I included only the briefest sketch of a proof of this proposition in [7]. This note is a response to several requests for more detail, and also mentions a couple of applications of the idea.

\section{THE MAIN RESULT}

Let $Z$ be a subset of a proper metric space $X$ and let $H$ be an ample $X$-module (i.e. a Hilbert space which is a "sufficiently large" module over

Date: January 5, 2018. 
$C_{0}(X)$, assumed fixed - I refer to [2] for terminology. The module action is denoted by $\rho: C_{0}(X) \rightarrow \mathfrak{B}(H)$.). The $C^{*}$-algebra $C^{*}(X)\left(\right.$ or $C^{*}(X ; H)$ if it is important to keep track of the particular Hilbert space) is then defined to be the norm closure of the controlled, locally compact operators on $H$, where we recall that a controlled (a.k.a. finite propagation) operator $T$ has the property that there is a constant $r$ for which

$$
d(\operatorname{Supp} \varphi, \operatorname{Supp} \psi)>r \Longrightarrow \rho(\varphi) T \rho(\psi)=0
$$

for all $\varphi, \psi \in C_{c}(X)$. A controlled operator $T$ is supported near $Z$ if there is another constant $r^{\prime}$ for which

$$
d(\operatorname{Supp} \varphi, Z)>r^{\prime} \Longrightarrow \rho(\varphi) T=0=T \rho(\varphi) .
$$

The norm closure of the set of controlled, locally compact operators supported near $Z$ is an ideal in $C^{*}(X)$, which we denote by $C^{*}(Z \subseteq X)$. It is easy to see [3] that the $K$-theory of $C^{*}(Z \subseteq X)$ is the same as that of $C^{*}(Z)$, if $Z$ is considered as a metric space in its own right.

Now we recall the relation of these concepts to index theory. Suppose that $X$ is actually a complete Riemannian manifold and that $S$ is a Clifford bundle, and let $H=L^{2}(X ; S)$ in forming the algebras above. The algebra $D^{*}(X)$ is defined to be the norm closure of the controlled, pseudolocal operators on $H$ : it is a unital $C^{*}$-algebra, and $C^{*}(X)$ is an ideal in it. The following key analytic lemma [2, Chapter 10] can be proved by the finite propagation speed method:

Lemma 2.1. Let $X$ be a complete Riemannian manifold, as above, and let $S$ be a Clifford bundle over it. Let $D$ denote the Dirac operator of $S$, considered as an unbounded, self-adjoint operator on $H=L^{2}(X ; S)$. If $f$ is a bounded continuous function on $\mathbb{R}$ that has finite limits at $\pm \infty$, then $f(D) \in D^{*}(X ; H)$. If $f$ tends to zero at $\pm \infty$, then $f(D) \in C^{*}(X ; H)$.

A normalizing function $\chi: \mathbb{R} \rightarrow[-1,1]$ is, by definition, a continuous, odd function that tends to \pm 1 at $\pm \infty$. Given such a function $\chi$, it follows from the preceding lemma that $\chi(D) \in D^{*}(X)$ and $\chi(D)^{2}-1 \in C^{*}(X)$. Moreover, if $\chi_{1}$ and $\chi_{2}$ are two normalizing functions, then it similarly follows that $\chi_{1}(D)-\chi_{2}(D) \in C^{*}(X)$. Thus the equivalence class of $\chi(D)$ gives a well-defined self-adjoint involution in $D^{*}(X) / C^{*}(X)$, defining an element $[\chi(D)] \in K_{j+1}\left(D^{*}(X) / C^{*}(X)\right)$ ( $j$ is determined by the grading of the operator - it is equal to the parity of $\operatorname{dim} X)$. Now we have

Definition 2.2. With the notation of Lemma 2.1, the coarse index of $D$ is

$$
\operatorname{Index}(D)=\partial[\chi(D)] \in K_{j}\left(C^{*}(X)\right),
$$

\footnotetext{
${ }^{1}$ It is denoted $C_{X}^{*}(Z)$ in [7], but the other notation now seems better to me.
} 
POSITIVE CURVATURE, PARTIAL VANISHING THEOREMS AND COARSE INDICES 3

where $\partial: K_{j+1}\left(D^{*}(X) / C^{*}(X)\right) \rightarrow K_{j}\left(C^{*}(X)\right)$ is the boundary map in the long exact sequence of $C^{*}$-algebra $K$-theory.

Now let $Z \subseteq X$ as above. The algebra $C^{*}(Z \subseteq X)$ is an ideal in $D^{*}(X)$ (not just in $C^{*}(X)$ ). To prove our result we will need to sharpen Lemma2.1 as follows:

Lemma 2.3. Let notation be as in Lemma 2.1 Suppose that the curvature operator $R=R_{D}$ that appears in the Weitzenbock formula for $D$,

$$
D^{2}=\nabla^{*} \nabla+R_{D}
$$

is uniformly positive outside $Z$, say $R_{x} \geqslant a^{2} I$ for $x \notin Z$. Then for any $f \in C_{c}(-a, a)$ we have $f(D) \in C^{*}(Z \subseteq X)$.

Suppose that this lemma has been proved. Then choose a normalizing function $\chi$ such that $\chi^{2}-1$ is supported in $(-a, a)$. According to Lemma 2.3, the equivalence class of $\chi(D)$ is a (well-defined) self-adjoint involution in $D^{*}(X) / C^{*}(Z \subseteq X)$. Following the construction above, we obtain a localized index

$$
\operatorname{Index}_{Z}(D) \in K_{j}\left(C^{*}(Z \subseteq X)\right)
$$

which maps to the previously defined $\operatorname{Index}(D)$ under the K-theory map induced by the inclusion $C^{*}(Z \subseteq X) \rightarrow C^{*}(X)$. The existence of this localized index is the precise content of [7, Proposition 3.11]; it implies the version of the result stated in the introduction. To state it precisely:

Theorem 2.4. Let $M$ be a complete Riemannian and let $D$ be a Diractype operator whose associated curvature endomorphism $R_{D}$ is uniformly positive outside a subset $Z$ of $M$. Then the construction above defines a localized coarse index

$$
\operatorname{Index}_{Z}(D) \in K_{j}\left(C^{*}(Z \subseteq X)\right)
$$

which maps to the coarse index $\operatorname{Index}(D) \in K_{j}\left(C^{*}(M)\right)$ under the $K$ theory map induced by the inclusion $C^{*}(Z \subseteq X) \rightarrow C^{*}(X)$. (Here $j$ is the parity of $\operatorname{dim} M$.

The rest of this section will give the proof of Lemma 2.3. In order to use the finite propagation speed method, we consider first the properties of functions $f$ that have compactly supported Fourier transforms.

Lemma 2.5. With notation as in Lemma 2.3. suppose that $f \in \mathcal{S}(\mathbb{R})$ is an even function and has Fourier transform $\hat{f}$ supported in $(-r, r)$. Let $\varphi \in C_{0}(X)$ have support disjoint from a $2 r$-neighborhood of $Z$. Then

$$
\|f(D) \rho(\varphi)\| \leqslant\|\varphi\| \sup \{|f(\lambda)|:|\lambda| \geqslant a\}
$$

and the same estimate applies to $\rho(\varphi) f(D)$. 
Proof. We use the Fourier cosine formula

$$
f(D)=\frac{1}{\pi} \int_{0}^{r} \hat{f}(t) \cos (t D) d t,
$$

remembering that $\hat{f}(t)$ vanishes for $t>r$. Now let $U_{n}=\{x \in X$ : $d(x, Z)>n r\}$, for $n=1,2$, and consider the unbounded, symmetric operator which is equal to $D^{2}$ with domain $C_{c}^{\infty}\left(U_{1}\right)$. This operator is bounded below by $a^{2} I$ and therefore it has a Friedrichs extension on the Hilbert space $L^{2}\left(U_{1} ; S\right)$ which is also bounded below (with the same bound) and which we shall denote by $E$.

A standard finite propagation speed argument shows that if $s$ is smooth and compactly supported in $U_{2}$ then

$$
\cos (t D) s=\cos (t \sqrt{E}) s, \quad \text { for } 0 \leqslant t \leqslant r .
$$

In particular, $\cos (t D) \rho(\varphi)=\cos (t \sqrt{E}) \rho(\varphi)$ for these values of $t$. Via the Fourier integral above, this implies that $f(D) M_{\varphi}=f(\sqrt{E}) M_{\varphi}$. But since the spectrum of $\sqrt{E}$ is bounded below by $a$,

$$
|f(\sqrt{E})| \leqslant \sup \{|f(\lambda)|:|\lambda| \geqslant a\},
$$

and this gives the desired estimate.

There is a version of Lemma 2.5 without the evenness hypothesis.

Lemma 2.6. With notation as above, suppose that $f \in \mathcal{S}(\mathbb{R})$ has Fourier transform $\hat{f}$ supported in $(-r, r)$. Let $\varphi \in C_{0}(X)$ have support disjoint from a $4 r$-neighborhood of $Z$. Then

$$
\|f(D) \rho(\varphi)\| \leqslant 2\|\varphi\| \sup \{|f(\lambda)|:|\lambda| \geqslant a\}
$$

and the same estimate applies to $\rho(\varphi) f(D)$.

Proof. If $f$ is even, this is a consequence of Lemma 2.5. If $f$ is odd, use the $C^{*}$-identity to write

$$
\|f(D) \rho(\varphi)\|^{2} \leqslant\|\rho(\bar{\varphi})\|\left\||f|^{2}(D) \rho(\varphi)\right\| .
$$

The function $g=|f|^{2}$ is even, belongs to $\mathcal{S}(R)$ and has Fourier transform supported in $(-2 r, 2 r)$. Thus, applying Lemma 2.5 to the function $g$,

$$
\left\||f|^{2}(D) \rho(\varphi)\right\| \leqslant\|\varphi\| \sup \left\{|f(\lambda)|^{2}:|\lambda| \geqslant a\right\}
$$

and so we obtain (on taking the square root)

$$
\|f(D) \rho(\varphi)\| \leqslant\|\varphi\| \sup \{|f(\lambda)|:|\lambda| \geqslant a\}
$$

which gives the desired result for odd $f$. The general result is obtained by writing $f$ as a sum of even and odd components (this decomposition accounts for the extra factor of 2 in the statement of Lemma 2.6). 
Using this, let us complete the proof of Lemma 2.3. Let $f$ be as in that lemma, and let $\varepsilon>0$ be given. There exists a smooth function $g$ with compactly supported Fourier transform such that $\sup \{|g(\lambda)-f(\lambda)|$ : $\lambda \in \mathbb{R}\}<\varepsilon$. In particular, $|g(\lambda)|<\varepsilon$ for $|\lambda|>a$. Let $r$ be such that $\operatorname{Supp}(\hat{g}) \subseteq(-r, r)$ and let $\psi: X \rightarrow[0,1]$ be a continuous function equal to 1 on a $4 r$-neighborhood of $Z$ and vanishing off a $5 r$-neighborhood of $Z$. Write

$$
\begin{aligned}
f(D)=\rho(\psi) & g(D) \rho(\psi)+ \\
+ & \rho(1-\psi) g(D) \rho(\psi)+g(D) \rho(1-\psi)+(f(D)-g(D)) .
\end{aligned}
$$

The first term is a locally compact operator supported near $Z$, the second and third terms have norm bounded by $2 \varepsilon$ by lemma 2.6, and the fourth term has norm bounded by $\varepsilon$ by the spectral theorem. Thus, $f(D)$ lies within $5 \varepsilon$ of a locally compact operator supported near $Z$. Since $\varepsilon$ is arbitrary, $f(D) \in C^{*}(Z \subseteq X)$, as was to be shown.

\section{VANISHING RESULTS}

As a consequence of the discussion above, if the curvature operator $R$ is uniformly positive outside $Z$, and if the $K$-theory map $K_{*}\left(C^{*}(Z)\right) \rightarrow$ $K_{*}\left(C^{*}(X)\right)$ is zero, then the index $\operatorname{Index}(D) \in K_{*}\left(C^{*}(X)\right)$ must vanish. The usual vanishing theorem establishes this result when $Z=\emptyset$ (i.e., when we have uniformly positive curvature on the whole of $M$ ), so we can regard these sort of results as a generalization where one allows a "small amount" of non-positive curvature.

For example, we have

Proposition 3.1. Let $M$ be a complete connected noncompact Riemannian manifold, and let $D$ be a Dirac-type operator whose associated curvature $R$ is uniformly positive outside a compact set. Then $\operatorname{Index}(D)=0$.

Proof. Let $K$ be a compact set outside which the curvature is uniformly positive, and let $Z$ be the union of $K$ and a geodesic ray from one of its points to infinity. The index of $D$ then lies in the image of $K_{*}\left(C^{*}(Z)\right) \rightarrow$ $K_{*}\left(C^{*}(X)\right)$ by the discussion above. But $Z$ is coarsely equivalent to $\mathbb{R}^{+}$, so $K_{*}\left(C^{*}(Z)\right)=0$.

For another example, imagine that we are in the situation of the "partitioned manifold index theorem" of [5]. So, let $M$ be a non-compact manifold that is partitioned by a compact hypersurface $N$, which (say) is spin and of non zero $\widehat{\mathcal{A}}$-genus, into two pieces $M^{+}$and $M^{-}$.

Proposition 3.2. A partitioned manifold as described above admits no complete metric that has uniformly positive scalar curvature on just one of the partition components $\left(M^{+}\right.$or $\left.M^{-}\right)$. 
Proof. Suppose $M$ has such a metric. Using the distance from $N$, construct a proper, coarse map $g: M \rightarrow \mathbb{R}$ that induces the given partition. By definition, the partitioned manifold index is

$$
g_{*}(\operatorname{Index} D) \in K_{1}\left(C^{*}(|\mathbb{R}|)\right)=\mathbb{Z},
$$

and the index theorem of [5] equates this to the $\widehat{\mathcal{A}}$-genus of $N$. Now suppose that $M$ has positive scalar curvature over $M^{+}$. Then by our main result, the coarse index factors through $K_{1}\left(C^{*}\left(M^{-} \subseteq M\right)\right)$. But considering the commutative diagram

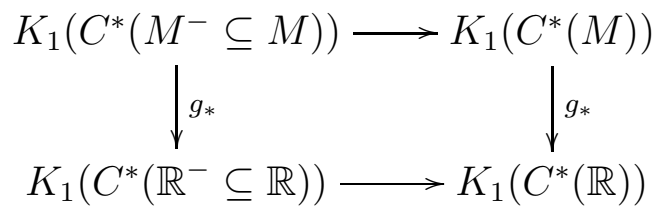

and noting that the bottom left-hand group is zero, we see that the coarse index vanishes.

\section{THE RELATIVE INDEX THEOREM}

The key technical result of [1, Chapter 4] is a relative index theorem which may be expressed as follows.

Suppose that $M_{1}$ and $M_{2}$ are complete Riemannian manifolds equipped with generalized Dirac operators $D_{1}$ and $D_{2}$ respectively, acting on (graded) Clifford bundles $S_{1}$ and $S_{2}$. Suppose further that these items agree near infinity: in other words, that there exist compact sets $Z_{i} \subseteq M_{i}$ an isometry $h: M_{1} \backslash Z_{1} \rightarrow M_{2} \backslash Z_{2}$ that is covered by a bundle isomorphism from $S_{1}$ to $S_{2}$, and that this isomorphism conjugates $D_{1}$ to $D_{2}$.

In these circumstances once can define a relative topological index $\operatorname{Index}_{r}\left(D_{1}, D_{2}\right) \in \mathbb{Z}$. There are several ways to define this quantity. For instance, one can compactify each of the $M_{i}$ identically outside $Z_{i}$ (thus obtaining compact manifolds $\widetilde{M}_{i}$ with elliptic operators $\widetilde{D}_{i}$ ) and then take the difference of the ordinary Fredholm indices, $\operatorname{Index}\left(\widetilde{D}_{1}\right)-\operatorname{Index}\left(\widetilde{D}_{2}\right)$, to define the relative index. Alternatively, one can take the Chern-Weil forms $\mathfrak{a}_{i}$ that are the representatives of the indices of $D_{i}$ according to the local index theorem, and "integrate their difference" over $M_{1} \cup M_{2}$ : specifically, note that $h^{*}$ takes $\mathfrak{a}_{2}$ to $\mathfrak{a}_{1}$, so that if we let $\mathfrak{a}$ be any smooth form on $M_{2}$, supported outside $Z_{2}$ and agreeing with $\mathfrak{a}_{2}$ near infinity, then the difference

$$
\int_{M_{1}}\left(\mathfrak{a}_{1}-h^{*} \mathfrak{a}\right)-\int_{M_{2}}\left(\mathfrak{a}_{2}-\mathfrak{a}\right)
$$

is well-defined (the integrands are compactly supported) and independent of the choice of $\mathfrak{a}$, and may be taken as the definition of the "integral of the difference of Chern-Weil forms". The equality of these two definitions of 
relative index is essentially Proposition 4.6 of [1]: it shows both that the first definition is independent of the choice of compactification, and that the second definition yields an integer.

Remark 4.1. Either definition implies that the relative index $\operatorname{Index}_{r}\left(D_{1}, D_{2}\right)$ depends only on the geometry of $M_{1}$ and $M_{2}$ (and the associated operators) in a neighborhood of the "regions of disagreement" $Z_{1}$ and $Z_{2}$. This stability property of the relative index is the basis for several calculations in [1].

Now suppose further that $D_{1}$ and $D_{2}$ have uniformly positive Weitzenbock curvature operators at infinity. Then $D_{1}$ and $D_{2}$, individually, are Fredholm operators, by Theorem 3.2 of [1] (a special case of our Theorem 2.4). The relative index theorem then states

Proposition 4.2. [1, Theorem 4.18] In the circumstances described above one has

$$
\operatorname{Index}\left(D_{1}\right)-\operatorname{Index}\left(D_{2}\right)=\operatorname{Index}_{r}\left(D_{1}, D_{2}\right) \text {. }
$$

We are going to generalize this result by allowing the "regions of disagreement" $Z_{i}$ to be non-compact. The first thing that we need to do is to define the relative index in this case. The following discussion, which is based on the ideas of [6], leads up to the generalized definition of the relative index, Definition 4.5 .

Let $M_{1}$ and $M_{2}$ be complete Riemannian manifolds (as above) and let $D_{1}$ and $D_{2}$ be generalized Dirac operators. Suppose that $M_{1}$ and $M_{2}$ are equipped with coarse maps $q_{1}$ and $q_{2}$ to a control space $X$ (a proper metric space), and that $Z$ is a subset of $X$. Put $Z_{i}=\overline{q_{i}^{-1}(Z)} \subseteq M_{i}$ for $i=1,2$. Suppose that there is a diffeomorphism $h: M_{1} \backslash Z_{1} \rightarrow M_{2} \backslash Z_{2}$ which is covered by an isomorphism of Clifford bundles and Dirac operators and which is compatible with the control maps in the sense that $q_{1}=q_{2} \circ f$.

From these data one can define a relative index in $K_{j}\left(C^{*}(Z)\right)$. Let $H_{i}$ be the Hilbert space $L^{2}\left(M_{i} ; S_{i}\right)$ and regard each $H_{i}$ as an $X$-module via the control map $q_{i}$. In this way we obtain translation algebras $C^{*}\left(X ; H_{i}\right)$, $i=1,2$, each of which contains an ideal $C^{*}\left(Z \subseteq X ; H_{i}\right)$ corresponding to $Z$. The isometry $h$ between the $M_{i}$ outside $Z_{i}$ passes to an unitary isomorphism $V$ between the $L^{2}\left(M_{i} \backslash Z_{i} ; S_{i}\right)$, and it is easy to see that conjugation by this unitary induces an isomorphism of quotient $C^{*}$-algebras

$$
\Phi: C^{*}\left(M_{1} ; H_{1}\right) / C^{*}\left(Z_{1} \subseteq M_{1} ; H_{1}\right) \rightarrow C^{*}\left(M_{2} ; H_{2}\right) / C^{*}\left(Z_{2} \subseteq M_{2} ; H_{2}\right) .
$$

Lemma 4.3. Let notation be as above and let $f \in C_{0}(\mathbb{R})$. Then

$$
\Phi\left[f\left(D_{1}\right)\right]=\left[f\left(D_{2}\right)\right],
$$

in the quotient algebra $C^{*}\left(M_{2} ; H_{2}\right) / C^{*}\left(Z_{2} \subseteq M_{2} ; H_{2}\right)$. 
There is also a " $D^{*}$-version" of this discussion. Namely, following the forthcoming PhD thesis of Paul Siegel [9] we can define ideals $D^{*}\left(Z_{i} \subseteq\right.$ $\left.M_{i} ; H_{i}\right)$ as the closure of the finite propagation, pseudolocal ${ }^{2}$ operators that are supported near $Z_{i}$ and are locally compact on $M_{i} \backslash Z_{i}$. Once again, conjugation by $U$ induces an isomorphism of quotient $C^{*}$-algebras

$$
\Psi: D^{*}\left(M_{1} ; H_{1}\right) / D^{*}\left(Z_{1} \subseteq M_{1} ; H_{1}\right) \rightarrow D^{*}\left(M_{2} ; H_{2}\right) / D^{*}\left(Z_{2} \subseteq M_{2} ; H_{2}\right) .
$$

Lemma 4.4. Let notation be as above and let $\chi$ be a normalizing function. Then

$$
\Psi\left[\chi\left(D_{1}\right)\right]=\left[\chi\left(D_{2}\right)\right]
$$

in the quotient algebra $D^{*}\left(M_{2} ; H_{2}\right) / D^{*}\left(Z_{2} \subseteq M_{2} ; H_{2}\right)$.

Proof. The proofs of both Lemmas 4.3 and 4.4 rely on the finite propagation speed method. First we give the proof for 4.3. Suppose that $f \in \mathcal{S}(\mathbb{R})$ and has Fourier transform $\hat{f}$ supported in $(-r, r)$. As usual, we write

$$
f(D)=\frac{1}{2 \pi} \int_{-\infty}^{\infty} \hat{f}(t) e^{i t D} d t
$$

and use the fact that, for a Dirac-type operator $D, e^{i t D}$ has propagation $|t|$. Let $\psi_{i}: M_{i} \rightarrow[0,1]$ be a smooth function equal to 1 on an $r$-neighborhood of $Z_{i}$ and vanishing off a $2 r$-neighborhood of $Z_{i}$, and such that $\psi_{1}=\psi_{2} \circ h$ on $M_{1} \backslash Z_{1}$. Write

$$
f\left(D_{i}\right)=f\left(D_{i}\right) \rho\left(\psi_{i}\right)+f\left(D_{i}\right)\left(1-\rho\left(\psi_{i}\right)\right) .
$$

Since $f\left(D_{i}\right)$ has propagation $r$, the first term belongs to $C^{*}\left(Z_{i} \subseteq H_{i}\right)$. By finite propagation speed we have

$$
V^{*} e^{i t D_{1}} \rho\left(1-\psi_{1}\right) V=e^{i t D_{2}} \rho\left(1-\psi_{2}\right) \quad \text { for }|t|<r .
$$

Consequently,

$$
V^{*} f\left(D_{1}\right)\left(1-\rho\left(\psi_{1}\right)\right) V=f\left(D_{2}\right)\left(1-\rho\left(\psi_{2}\right)\right)
$$

and the proof is complete for $f$ having compactly supported Fourier transform. The general result follows, since such $f$ are norm-dense in $C_{0}(\mathbb{R})$.

The proof of Lemma 4.4 follows a similar pattern, where the Fourier transform $\hat{\chi}$ must now be understood as a distribution with a mild singularity at 0 . The only additional argument that is needed is to show that

$$
\left(V \chi\left(D_{1}\right) V^{*}-\chi\left(D_{2}\right)\right) \rho(\varphi)
$$

is compact for $\varphi \in C_{0}\left(M_{2} \backslash Z_{2}\right)$. Suppose in fact that $\varphi$ is compactly supported. Then there is a constant $r>0$ such that $d\left(Z_{2}, \operatorname{Supp}(\varphi)\right)>$ $r$ and, if we should choose the normalizing function $\chi$ to have Fourier

\footnotetext{
"Finite propagation" is defined with respect to the control space $X$ via the control maps $q_{i}$; "pseudolocal" is defined with respect to the ambient manifold $M_{i}$.
} 
transform supported in $(-r, r)$, then finite propagation speed shows that the displayed quantity in 4.1 is not just compact - it is actually zero! The general case follows from this particular one, since any two normalizing functions differ by some $g \in C_{0}(\mathbb{R})$, and we already know that for such $g$, the individual terms $g\left(D_{1}\right)$ and $g\left(D_{2}\right)$ are locally compact.

Now let $\pi_{i}$ denote the quotient map $C^{*}\left(M_{i}\right) \rightarrow C^{*}\left(M_{i}\right) / C^{*}\left(Z_{i} \subseteq M_{i}\right)$ or $D^{*}\left(M_{i}\right) \rightarrow D^{*}\left(M_{i}\right) / D^{*}\left(Z_{i} \subseteq M_{i}\right)$ as appropriate. Let us define $\bar{A}$ to be the pull-back $C^{*}$-algebra

$$
A=\left\{\left(T_{1}, T_{2}\right) \in C^{*}\left(M_{1} ; H_{1}\right) \oplus C^{*}\left(M_{2} ; H_{2}\right): \Phi\left(\pi_{1}\left(T_{1}\right)\right)=\pi_{2}\left(T_{2}\right)\right\} .
$$

Similarly define $B$ to be the pull-back $C^{*}$-algebra

$$
B=\left\{\left(T_{1}, T_{2}\right) \in D^{*}\left(M_{1} ; H_{1}\right) \oplus D^{*}\left(M_{2} ; H_{2}\right): \Phi\left(\pi_{1}\left(T_{1}\right)\right)=\pi_{2}\left(T_{2}\right)\right\} .
$$

Then $A$ is an ideal in $B$. Let $D$ denote the Dirac operator on the disjoint union $M_{1} \sqcup M_{2}$. Lemmas 4.3 and 4.4 above show that for a normalizing function $\chi$, the operator $\chi(D)$ is an element of $B$, and that for a function $f \in C_{0}(\mathbb{R})$, the operator $f(D)$ is an element of the ideal $A$. Consequently there is defined an index of $D$

$$
\operatorname{Index}_{Z}(D) \in K_{j}(A) \text {. }
$$

The group $K_{j}(A)$ can be decomposed as a direct sum. In fact, let $U: H_{1} \rightarrow$ $\mathrm{H}_{2}$ be a covering isometry for the identity map [2, Definition 6.3.9] that agrees on $L^{2}\left(M_{1} \backslash Z_{1}\right)$ with the isomorphism $L^{2}\left(M_{1} \backslash Z_{1}\right) \rightarrow L^{2}\left(M_{2} \backslash Z_{2}\right)$ induced by $h$. (The hypothesis that $h$ boundedly commutes with the control maps assures the existence of such an isometry.) Then there is a split short exact sequence

$$
0 \rightarrow C^{*}\left(Z_{1} \subseteq M_{1}\right) \rightarrow A \rightarrow C^{*}\left(M_{2}\right) \rightarrow 0,
$$

where the first map is $a \mapsto(a, 0)$, the second is $\left(a_{1}, a_{2}\right) \mapsto a_{2}$, and the splitting maps $a$ to $\left(U^{*} a U, a\right)$. From this split short exact sequence we obtain a direct sum decomposition

$$
K_{j}(A)=K_{j}\left(C^{*}\left(Z_{1} \subseteq M_{1}\right)\right) \oplus K_{j}\left(C^{*}\left(M_{2}\right)\right) .
$$

Definition 4.5. The relative index of the above data is the component in $K_{j}\left(C^{*}\left(Z_{1} \subseteq M_{1}\right)\right)=K_{j}\left(C^{*}(Z)\right)$ of $\operatorname{Index}_{Z}(D) \in K_{j}(A)$. We denote it by $\operatorname{Index}_{r}\left(D_{1}, D_{2}\right)$.

The generalization of Gromov-Lawson's relative index theorem is then

Theorem 4.6. Let $\left(M_{i}, D_{i}, q_{i}\right)$ be a set of relative-index data over $(X, Z)$, with the notation described above. Suppose that the operators $D_{i}$ have uniformly positive Weitzenbock curvature operators outside $Z_{i}$. Then each 
$D_{i}$ has a localized coarse index in $K_{j}\left(C^{*}(Z)\right)$, by Theorem 2.4 and the identity

$$
\operatorname{Index}_{Z}\left(D_{1}\right)-\operatorname{Index}_{Z}\left(D_{2}\right)=\operatorname{Index}_{r}\left(D_{1}, D_{2}\right)
$$

holds in $K_{j}\left(C^{*}(Z)\right)$.

(The case considered by Gromov and Lawson can be recovered by taking $X=\mathbb{R}^{+}, Z=\{0\}$.)

Proof. Let $A$ be the pull-back algebra that we introduced in our definition of the relative index (so that $A$ consists of pairs $\left(T_{1}, T_{2}\right), T_{i} \in C^{*}\left(M_{i}\right)$, that "agree away from $Z$ ".) Let $J$ be the ideal in $A$ that consists of pairs $\left(T_{1}, T_{2}\right)$ where each $T_{i}$ belongs to $C^{*}\left(Z_{i} \subseteq M_{i}\right)$; in fact, $J$ is simply the direct sum $C^{*}\left(Z_{1} \subseteq M_{1}\right) \oplus C^{*}\left(Z_{2} \subseteq M_{2}\right)$. Let $D$ denote the Dirac operator on $M_{1} \sqcup M_{2}$.

Because of the positive curvature away from $Z$ it follows from Lemma2.3 that, for $f \in C_{0}(\mathbb{R}), f(D)$ belongs to the ideal $J$. Thus, in this case, the index $\operatorname{Index}_{Z}(D)$ defined in Equation 4.2 in fact belongs to $K_{j}(J)=$ $K_{j}\left(C^{*}(Z)\right) \oplus K_{j}\left(C^{*}(Z)\right)$,] and it is apparent from the definitions that, in terms of this direct sum decomposition,

$$
\operatorname{Index}_{Z}(D)=\left(\operatorname{Index}_{Z}\left(D_{1}\right), \operatorname{Index}_{Z}\left(D_{2}\right)\right) \text {. }
$$

The definition of the relative index tells us to take the component of $\operatorname{Index}_{Z}(D)$ in $K_{j}\left(C^{*}(Z)\right)$ in the direct sum decomposition coming from the split short exact sequence 4.3 . Restricted to $J$, this sequence takes the form

$$
0 \rightarrow C^{*}(Z) \rightarrow C^{*}(Z) \oplus C^{*}(Z) \rightarrow C^{*}(Z) \rightarrow 0,
$$

where the first map is inclusion on the first factor, the second is projection on the second factor, and the splitting used is $a \mapsto(a, a)$. Using this splitting, one finds that the relevant component of $\operatorname{Index}_{Z}(D)=$ $\left(\operatorname{Index}_{Z}\left(D_{1}\right), \operatorname{Index}_{Z}\left(D_{2}\right)\right)$ is $\operatorname{Index}_{Z}\left(D_{1}\right)-\operatorname{Index}_{D}\left(Z_{2}\right)$, as required.

As we observed above, it is an important feature of the Gromov-Lawson relative index that it depends only on the geometry of a neighborhood of the "region of disagreement". The corresponding result is also true in our more general context, and is a key to the applications of the relative index concept in [7].

Proposition 4.7. [7, Theorem 3.12] The relative index of Definition 4.5 depends only on the geometry of a metric neighborhood of $Z_{1}$ and $Z_{2}$ and the operators thereon.

Notice that this statement is independent of any positive-curvature hypotheses. 
Proof. This follows from the results of [8]. In that paper, it is shown that to a set of relative index data (as described in this section), one may associate a relative homology class that lies in the $K$-homology group $K_{*}(Z)$. Moreover, comparison of the definitions shows that our coarse relative index is simply the image of this relative homology class under the coarse assembly map

$$
A: K_{*}(Z) \rightarrow K_{*}\left(C^{*}(Z)\right) .
$$

The result is therefore a consequence of Proposition 4.8 of [8], which states that in fact the relative homology class of a set of relative index data depends only on the geometry in a neighborhood of the region of disagreement.

\section{REFERENCES}

[1] M. Gromov and H.B. Lawson. Positive scalar curvature and the Dirac operator. Publications Mathématiques de l'Institut des Hautes Études Scientifiques, 58:83-196, 1983.

[2] N. Higson and J. Roe. Analytic K-Homology. Oxford Mathematical Monographs. Oxford University Press, Oxford, 2000.

[3] N. Higson, J. Roe, and G. Yu. A coarse Mayer-Vietoris principle. Mathematical Proceedings of the Cambridge Philosophical Society, 114:85-97, 1993.

[4] A. Lichnerowicz. Spineurs harmoniques. Comptes Rendus de l'Académie des Sciences de Paris, 257:7-9, 1963.

[5] J. Roe. Partitioning non-compact manifolds and the dual Toeplitz problem. In D. Evans and M. Takesaki, editors, Operator Algebras and Applications, pages 187-228. Cambridge University Press, Cambridge, 1989.

[6] J. Roe. A note on the relative index theorem. Quarterly Journal of Mathematics, 42:365-373, 1991.

[7] J. Roe. Index Theory, Coarse Geometry, and the Topology of Manifolds, volume 90 of CBMS Conference Proceedings. American Mathematical Society, Providence, R.I., 1996.

[8] John Roe and Paul Siegel. Sheaf theory and Paschke duality. Preprint, 2012. http://arxiv.org/abs/1210.6420

[9] Paul Siegel. Homological calculations with analytic structure groups. PhD thesis, Penn State, 2012.

Department of Mathematics, Penn State University, University Park PA 16802 